\title{
SUBLINEAR DIFFERENTIAL POLYNOMIALS
}

\author{
BY \\ D. G. MEAD
}

Introduction $\left({ }^{1}\right)$. Let $c$ be a complex number. The general solution of the differential equation

$$
c y y^{\prime \prime}-(c-1) y^{\prime 2}=0
$$

consists of all functions of the form $a(x+b)^{c}$ where $a$ and $b$ are constants. If $c$ is not a natural number then, as is easy to see, there does not exist a linear differential equation admitting all these functions as solutions; on the other hand, if $c$ is a natural number then the equation $y^{(c+1)}=0$ admits all these functions as solutions, but no homogeneous linear differential equation of lower order does.

Thus, there arises the following question. Given an irreducible differential polynomial $F$ (in one differential indeterminate $y$, and with coefficients in some ordinary differential field $\mathcal{F}$ of characteristic zero), does there exist a homogeneous linear differential equation $L=0$ the general solution of which contains the general solution of the differential equation $F=0$ and, if such linear equations do exist, of how low an order? A complete answer to this question does not seem to be easy. Indeed, the above example shows that the answer is not determined by the knowledge of the monomials which appear in $F$ with nonzero coefficients; some knowledge of the coefficients themselves is necessary.

In this paper we describe various conditions under which the answer is negative. Actually, it is convenient to treat a slightly broader question. Let $F$ be an irreducible element of the differential polynomial ring $\mathcal{F}\{y\}$ with $F \notin \mathcal{F}$, and let $n=\operatorname{ord} F, S$ be the separant of $F$ (that is, $\left.S=\partial F / \partial y^{(n)}\right)$, and $I$ be the initial of $F$ (that is, the coefficient of the highest power of $y^{(n)}$ appearing in $F$ ). If $G \in \mathfrak{F}\{y\}$ then each of the following three conditions is necessary and sufficient for $G$ to vanish on the general solution of $F=0$ : (i) $G$ is contained in the perfect differential ideal $\{F\}: S$ of $\mathcal{F}\{y\}$; (ii) $G$ is contained in the differential ideal $[F]: S^{\infty}\left(=\bigcup_{1 \leq j<\infty}[F]: S^{j}\right)$; (iii) there exists a nonzero differential polynomial $B \in \mathcal{F}\{y\}$ of order $<n$ such that $B G \in\{F\}$. If we relinquish the condition that $F$ be irreducible, supposing merely that $F \notin \mathcal{F}$, than these three conditions are no longer equivalent. We define $F$ to be sublinear (of type $r$ ) if there exists a nonzero homogeneous linear $L \in \mathfrak{F}\{y\}$ (or order $r$ ) satisfying the condition (iii) on $G$, that is, if every solution of

Received by the editors April 11, 1959.

(1) Some familiarity with the basic concepts, terminology and notation of differential algebra will be assumed. See Ritt [2]. 
$F=0$ is a solution of either the linear equation $L=0$ or the lower order equation $B=0$ (and there exists no such $L$ of order $<r$ ). It is clear that $F$ is sublinear if and only if the product $F_{0}$ of its distinct irreducible factors of order $n$ is sublinear, so we may assume that $F$ has no multiple factors of order $n$. Under this assumption, the three conditions above are again equivalent.

As defined, the condition that $F$ be sublinear of type $r$ is a relative one, depending on the differential field $\mathcal{F}$. However, it is easy to see by a linear independence argument that if $F$ is sublinear relative to some differential field $\mathcal{S} \mathcal{F}$ then $F$ is sublinear relative to $\mathcal{F}$. Therefore we may suppose given, once and for all, an ordinary differential field $\mathfrak{u}$ which is a universal extension $\left(^{2}\right)$ of the field of rational numbers, and then work entirely within the differential polynomial ring $\mathfrak{u}\{y\}$; we shall denote the field of constants of $u$ by $\varkappa$.

In Chapters I and II we suppose that $F$ is nonlinear and has degree in $y^{(n)}$ equal to 1 (that is, $S=I$ ). In Chapter I, after introducing certain gradings of $u\{y\}$, called $F$-gradings, we show (Lemma 8 ) that if $F$ is sublinear then so are certain differential polynomials $G \in \mathcal{K}\{y\}$ related to $F$ in a certain way by means of an $F$-grading. In Chapter II we show that $F$ is not sublinear when $\operatorname{deg} I=0$ and for certain cases when $\operatorname{deg} I \neq 0$. In Chapter III, we relinquish the special hypothesis on $F$. The main result (Theorem 6 ) is that if $F$ is not linear then there is a natural number $k$ such that the $i$ th derivative $F^{(i)}$ is not sublinear for $i \geqq k$. Theorem 5 shows that for "most" $F$ we may take $k=2$.

If $F^{\prime}=S \cdot G$ where $G$ is linear, then $F^{\prime}$ is obviously sublinear. We conjecture that this is the only time that $F^{\prime}$ is sublinear, though the proof (if the statement is correct) probably requires new methods.

The universal extension field $\mathcal{u}$ contains a solution of the differential equation $y^{\prime}=1$. We fix a solution of this equation and denote it by $x$. It is easy to see that $x$ is transcendental over $\nVdash$.

I wish to take this opportunity to thank Professor E. R. Kolchin for the numerous valuable suggestions and criticisms that he has given me, without which this paper would not have been possible.

\section{Chapter I. Preliminaries}

In this chapter $F$ denotes any nonlinear differential polynomial $I y^{(n)}+Q$ with $I, Q \in \mathcal{u}\{y\}, I Q \neq 0, \operatorname{deg} Q>0$, and ord $I Q<n$.

1. Types of gradings. For any sequence $g=\left(\alpha_{j}\right)_{j \in N}$ of real numbers $\left({ }^{3}\right)$, the set of monomials $y^{i_{0}} y^{\prime i_{1}} \ldots y^{(v) i_{v}}$ for which $\sum i_{j} \alpha_{j}$ has a given value $s$, generates a vector space $R_{s}$ over $\mathcal{U}$, and $\mathcal{U}\{y\}$ is the direct sum of all the spaces $R_{s}$; obviously $R_{s} R_{t} \subset R_{s+t}$ so that there is defined a grading of $u\{y\}$. We denote this grading by the same letter $g$. We call the elements of $R_{\varepsilon} g$-homo-

(2) The definition and existence proof of a universal extension are given in Kolchin [1].

(3) $N$ will be used exclusively to denote the set of natural numbers. 
geneous of grade $s$. An element $P$ of $u\{y\}$ can be written in a unique way in the form $P=\sum P_{s}$, where $P_{s} \in R_{s}$ for each real number $s$, and $P_{s} \neq 0$ for only finitely values of $s$; we call $P_{s}$ the g-homogeneous part of $P$ of grade $s$. If $P \neq 0$, the largest real $s$, such that $P_{\mathrm{s}} \neq 0$ will be called the grade of $P$ and will be denoted by $g(P)$; if $s=g(P)$, then we call $P_{s}$ the highest part of $P$ and denote it by $P^{*}$. As an example we may consider the grading $\left(\alpha_{j}\right)_{j \in N}$ in which $\alpha_{j}=j(j \in N)$; in this case $g(P)$ is called the weight of $P$ and is denoted by wtP.

A grading $g$ of $u\{y\}$ defined as above will be called arithmetic if: (a) $g\left(y^{(i)}\right)$ $\geqq 0$ for every $i$; (b) there exists a non-negative integer $q$ and a real number $\Delta>0$ such that

$$
g\left(y^{(i+1)}\right)-g\left(y^{(i)}\right) \begin{cases}<\Delta & (0 \leqq i<q), \\ =\Delta & (i \geqq q) .\end{cases}
$$

The numbers $q$ and $\Delta$, which are obviously unique, will be called the order of $g$ and the difference of $g$, respectively, and will be denoted by ord $g$ and diff $g$, respectively. The weight is an arithmetic grading of order 0 and difference 1 .

LEMMA 1. Let $g$ be an arithmetic grading of $u\{y\}$ of order $q$ and difference $\Delta ;$ let $P \in \mathcal{U}\{y\}$ and $P \notin \mathcal{U}$. Then $g\left(P^{\prime}\right)<g(P)+\Delta$ if ord $P^{*}<q$, and $g\left(P^{\prime}\right)$ $=g(P)+\Delta$ if ord $P^{*}>q$.

Proof. Obvious.

2. Semi- $F$-gradings and $F$-gradings. An arithmetic grading of $u\{y\}$ will be called a semi-F-grading if $g\left(I y^{(n)}\right) \leqq g(Q)$ and ord $Q^{*} \geqq$ ord $g$. If in addition $g\left(I y^{(n)}\right)=g(Q)$, then $g$ will be called an $F$-grading. It is obvious that if $g$ is a semi- $F$-grading then ord $g \leqq n-1$.

LEMMA 2. If $g=\left(\alpha_{i}\right)_{i \in N}$ is a semi-F-grading of order $q$, which is not an $F$ grading, there exists a semi-F-grading $g^{\prime}$ such that ord $g^{\prime} \geqq$ ord $g$ and diff $g^{\prime}$ $>$ diff $g$; more precisely ord $g^{\prime}=$ ord $Q^{*}$.

Proof. Let $k$ be any natural number; for any monomial $P=y^{i_{0}} y^{\prime i_{1}} \cdots y^{(v) i v}$ define $w(P, k)=\sum_{j=k}^{\nu} i_{j}(j-k)$; for any nonzero differential polynomial $G$ in $y$ define $w(G, k)$ as the maximum $w(P, k)$ where $P$ ranges over the monomials effectively present in $G$. Let $r=$ ord $Q^{*}$. (Note that $r \geqq q$.) Let

$$
\alpha_{i}^{\prime}=\left\{\begin{array}{l}
\alpha_{i}, \quad(0 \leqq i<r), \\
\alpha_{i}+\frac{g(Q)-g\left(I y^{(n)}\right)}{w\left(I y^{(n)}, r\right)}(i-r), \quad(i \geqq r) .
\end{array}\right.
$$

It is clear that $g^{\prime}=\left(\alpha_{i}^{\prime}\right)$ is an arithmetic grading with ord $g^{\prime}=r \geqq q=$ ord $g$ and diff $g^{\prime}=\operatorname{diff} g+\left(g(Q)-g\left(I y^{(n)}\right)\right) /\left(w\left(I y^{(n)}, r\right)\right)>\operatorname{diff} g . g^{\prime}$ is a semi- $F$-grading since 


$$
\begin{aligned}
g^{\prime}\left(I y^{(n)}\right) & =\max g^{\prime}(P) \leqq \max g(P)+\max w(P, r) \cdot \frac{g(Q)-g\left(I y^{(n)}\right)}{w\left(I y^{(n)}, r\right)} \\
& =g\left(I y^{(n)}\right)+g(Q)-g\left(I y^{(n)}\right)=g(Q) \leqq g^{\prime}(Q),
\end{aligned}
$$

where $P$ ranges over the monomials which are effectively present in $I y^{(n)}$.

LEMMA 3. If there exists no semi-F-grading of order $>q$, and if $g=\left(\alpha_{i}\right)$ and $g^{\prime}=\left(\alpha_{i}^{\prime}\right)$ are two semi-F-gradings of order $q$ with $\alpha_{i}=\alpha_{i}^{\prime}$ for all $i \leqq q$, then $g(Q)=g^{\prime}(Q)$.

Proof. Assume $g(Q)>g^{\prime}(Q)$. Since $\alpha_{i}=\alpha_{i}^{\prime}$ for $i=1, \cdots, q$, the maximum order $r$ of terms $P$ effectively present in $Q$ with $g(P)=g(Q)$ must be greater than $q$. By Lemma 2 , there exists a semi- $F$-grading of order $r>q$, contrary to hypothesis.

The two preceding lemmas can be combined to show the following relationship between semi- $F$-gradings and $F$-gradings.

Lemma 4. If there exists a semi-F-grading of order $q$, there exists an $F$ grading of order $\geqq q$.

Proof. The order of every semi- $F$-grading being less than $n$, there exists an integer $r \geqq q$ such that there is a semi-F-grading of order $r$ but none of order $>r$. Let $g=\left(\alpha_{i}\right)$ be a fixed semi- $F$-grading of order $r$.

We shall see that there exists a real number $m$ such that, if $g^{\prime}=\left(\alpha_{i}^{\prime}\right)$ is any semi- $F$-grading of order $r$ with $\alpha_{i}^{\prime}=\alpha_{i}$ for all $i \leqq r$, then $\operatorname{diff} g^{\prime} \leqq m$. Indeed from Lemma 3, $g^{\prime}(Q)=g(Q)$; if diff $g^{\prime}$ could be arbitrarily large, $g^{\prime}\left(I y^{(n)}\right)$ would be large, contradicting the fact that $g^{\prime}$ is a semi- $F$-grading, i.e. $g^{\prime}\left(I y^{(n)}\right)$ $\leqq g^{\prime}(Q)$.

Let $\Delta$ be the least upper bound of the differences of all such semi- $F$-gradings $g^{\prime}$. Then

$$
\beta_{i}=\left\{\begin{array}{l}
\alpha_{i}, \quad(0 \leqq i<r), \\
\alpha_{i}+(i-r) \Delta, \quad(i \geqq r),
\end{array}\right.
$$

will prove to be the desired $F$-grading. It is clear that $\left(\beta_{i}\right)$ is a semi- $F$-grading. If it were not an $F$-grading, the construction of Lemma 2 would provide a semi- $F$-grading of even larger difference (not affecting the $\beta_{i}$ with $i \leqq r$ ) contrary to the maximal condition of $\Delta$. The proof of the lemma is complete.

3. Basic lemma. For each natural number $m$, define a mapping $\delta_{m}: \mathcal{u}\{y\} \rightarrow \mathfrak{U}\{y\}$ by the formula:

$$
\begin{aligned}
\delta_{m}(P)=I & {\left[I\left(P^{\prime}-y^{(n)} \frac{\partial P}{\partial y^{(n-1)}}\right)-Q \frac{\partial P}{\partial y^{(n-1)}}\right] } \\
& -m P\left[I\left(I^{\prime}-y^{(n)} \frac{\partial I}{\partial y^{(n-1)}}\right)-Q \frac{\partial I}{\partial y^{(n-1)}}\right] .
\end{aligned}
$$


Lemma 5. If $I^{m} y^{(r)} \equiv P(\bmod [F])$, then $I^{m+2} y^{(r+1)} \equiv \delta_{m} P(\bmod [F])$.

Proof. If $I^{m} y^{(r)} \equiv P$, then $I^{m} y^{(r+1)}+m I^{\prime} I^{m-1} y^{(r)} \equiv P^{\prime}$ so that $I^{m+2} y^{(r+1)}$ $+m I I^{\prime} I^{m} y^{(r)} \equiv I^{2} P^{\prime}$; thus

$$
\begin{aligned}
I^{m+2} y^{(r+1)}+m I I^{\prime} P & \equiv I\left[I\left(P^{\prime}-y^{(n)} \frac{\partial P}{\partial y^{(n-1)}}\right)+I y^{(n)} \frac{\partial P}{\partial y^{(n-1)}}\right] \\
& \equiv I\left[I\left(P^{\prime}-y^{(n)} \frac{\partial P}{\partial y^{(n-1)}}\right)-Q \frac{\partial P}{\partial y^{(n-1)}}\right]
\end{aligned}
$$

whence

$$
\begin{aligned}
I^{m+2} y^{(r+1)} \equiv I & {\left[I\left(P^{\prime}-y^{(n)} \frac{\partial P}{\partial y^{(n-1)}}\right)-Q \frac{\partial P}{\partial y^{(n-1)}}\right] } \\
& -m P\left[I\left(I^{\prime}-y^{(n)} \frac{\partial I}{\partial y^{(n-1)}}\right)+I y^{(n)} \frac{\partial I}{\partial y^{(n-1)}}\right] \equiv \delta_{m} P .
\end{aligned}
$$

CoRollary. $I^{2 t+1} y^{(n+t)} \equiv-\delta_{2 t-1} \cdots \delta_{3} \delta_{1} Q(\bmod [F])$.

Lemma 6. Let $g$ be an F-grading, and let $P \in \mathcal{U}\{y\}$. Then $g\left(\delta_{m} P\right) \leqq g(P)$ $+g\left(I^{2}\right)+\operatorname{diff} g$.

Proof. Obvious from the definition of $\delta_{m}$.

For any $F$-grading $g$ and any natural number $m$, define a mapping $\delta_{g, m}: \mathfrak{U}\{y\} \rightarrow \mathfrak{U}\{y\}$ by the formula:

$$
\begin{aligned}
\delta_{g, m} P=I^{*} & \left(I^{*} \sum_{j=\mathrm{ord}}^{n-2} y^{(j+1)} \frac{\partial P}{\partial y^{(j)}}-Q^{*} \frac{\partial P}{\partial y^{(n-1)}}\right) \\
& -m P\left(I^{*} \sum_{j=\mathrm{ord} g}^{n-2} y^{(j+1)} \frac{\partial I^{*}}{\partial y^{(j)}}-Q^{*} \frac{\partial I^{*}}{\partial y^{(n-1)}}\right) .
\end{aligned}
$$

Lemma 7. Let $g$ be an $F$-grading and let $P \in \mathcal{U}\{y\}$ be g-homogeneous and of order $\geqq$ ord $g$. Then $\delta_{o, m} P$ is $g$-homogeneous of grade $g(P)+g\left(I^{2}\right)+\operatorname{diff} g$.

Proof. Obvious from the definition of $\delta_{g, m}$.

Lemma 8 (BASIC Lemma). Let $g$ be an F-grading and let $t$ be a natural number.

(a) If $F$ is sublinear of type $\leqq n+t$, then $\delta_{g, 2 t-1} \cdots \delta_{g, 3} \delta_{g, 1} Q^{*}=0$.

(b) Let a be the algebra over $\nVdash$ generated by the coefficients in $F^{*}$ and all the derivatives $y^{(i)}(0 \leqq i<\infty)$, znd let $\gamma: Q \rightarrow \mathcal{U}\{y\}$ be an algebra homomorphism mapping each coefficient in $F^{*}$ into $K$, mapping each $y^{(i)}$ with $i<$ ord $g$ into $\Re$, and mapping each $y^{(i)}$ with $i \geqq$ ord $g$ onto itself, such that $\gamma\left(I^{*} Q^{*}\right) \neq 0$. If

$$
\delta_{g, 2 t-1} \cdots \delta_{g, s} \delta_{g, 1} Q^{*}=0 \text { then } y^{(n+t)} \in\left\{\gamma\left(F^{*}\right)\right\} ; \gamma\left(I^{*}\right) .
$$


Proof. (a) Let $\sum_{i=0}^{n+t} a_{i} y^{(i)} \in\{F\}: I$ with $a_{i} \in \mathcal{U}$ and $a_{n+t} \neq 0$. Then

$$
I^{2 t+1} \sum_{i=0}^{n-1} a_{i} y^{(i)}+\sum_{j=0}^{t} a_{n+j} I^{2 t-2 j} I^{2 j+1} y^{(n+j)}=I^{2 t+1} \sum_{i=0}^{n+t} a_{i} y^{(i)} \in\{F\}: I,
$$

so, by the corollary to Lemma 5 , the differential polynomial

$$
H=I^{2 t+1} \sum_{i=0}^{n-1} a_{i} y^{(i)}-\sum_{j=0}^{t} a_{n+j} I^{2 t-2 j} \delta_{2 j-1} \cdots \delta_{3} \delta_{1} Q
$$

is in $\{F\}: I$. As $H$ is reduced with respect to $F, H=0$; therefore the $g$-homogeneous part of $H$ of grade $h=g(Q)+t\left[g\left(I^{2}\right)+\operatorname{diff} g\right]$ is zero. Now, $g\left(I^{2 t+1} \sum_{i=0}^{n-1} a_{i} y^{(i)}\right) \leqq(2 t+1) g(I)+g\left(y^{(n-1)}\right)=g\left(I y^{(n)}\right)+\operatorname{tg}\left(I^{2}\right)-\operatorname{diff} g<h$; also, by Lemma $6, g\left(\sum_{j=0}^{t-1} a_{n+j} I^{2 t-2 j} \delta_{2 j-1} \cdots \delta_{1} Q\right) \leqq g(Q)+(t-1)\left[g\left(I^{2}\right)+\right.$ diff $\left.g\right]$ $+g\left(I^{2}\right)<h$; furthermore, if $r<g(Q)$ and $Q_{r}$ denotes the $g$-homogeneous part of $Q$ of grade $r$, then, again by Lemma $6, g\left(a_{n+t} \delta_{2 t-1} \cdots \delta_{3} \delta_{1} Q_{r}\right)<h$. Therefore the the $g$-homogeneous part of $H$ of grade $h$ equals the $g$-homogeneous part of $-a_{n+t} \delta_{2 t-1} \cdots \delta_{3} \delta_{1} Q^{*}$ of grade $h$. By Lemma 1 , this in turn equals the $g$ homogeneous part of $-a_{n+t} \delta_{g, 2 t-1} \cdots \delta_{g, 1} Q^{*}$ of grade $h$, which, by Lemma 5, coincides with $-a_{n+t} \delta_{g, 2 t-1} \cdots \delta_{g, 1} Q^{*}$ itself. Since $a_{n+t} \neq 0$, it follows that $\delta_{g, 2 t-1} \cdots \delta_{g, 1} Q^{*}=0$.

(b) If $P \in Q$ then obviously $\delta_{g, m} P \in Q$, and $\gamma\left(\delta_{g, m} P\right)=\delta_{m}^{\prime}(\gamma(P))$, where $\delta_{m}^{\prime}$ is the mapping of $u\{y\}$ into $u\{y\}$ defined by the formula

$$
\begin{aligned}
\delta_{m}^{\prime} A= & \gamma\left(I^{*}\right)\left[\gamma\left(I^{*}\right)\left(A^{\prime}-y^{(n)} \frac{\partial A}{\partial y^{(n-1)}}\right)-\gamma\left(Q^{*}\right) \frac{\partial A}{\partial y^{(n-1)}}\right] \\
& -m A\left[\gamma\left(I^{*}\right)\left(\left(\gamma\left(I^{*}\right)\right)^{\prime}-y^{(n)} \frac{\partial\left(\gamma\left(I^{*}\right)\right)}{\partial y^{(n-1)}}\right)-\gamma\left(Q^{*}\right) \frac{\partial \gamma\left(I^{*}\right)}{\partial y^{(n-1)}}\right] .
\end{aligned}
$$

It follows that $\delta_{2 t-1}^{\prime} \cdots \delta_{3}^{\prime} \delta_{1}^{\prime}\left(Q^{*}\right)=\gamma\left(\delta_{g, 2 t-1} \cdots \delta_{g, 3} \delta_{g, 1} Q^{*}\right)=0$. By the corollary to Lemma 5 (applied to $\gamma\left(F^{*}\right)=\gamma\left(I^{*}\right) y^{(n)}+\gamma\left(Q^{*}\right)$ instead of $F=I y^{(n)}+Q$ ), this implies that $\gamma\left(I^{*}\right)^{2 t+1} y^{(n+t)} \equiv 0 \bmod \left[\gamma\left(F^{*}\right)\right]$ so that $y^{(n+t)} \in\left\{\gamma\left(F^{*}\right)\right\}: \gamma\left(I^{*}\right)$.

Chapter II. Differential polynomials, $F=I y^{(n)}+Q$

In this chapter $F$ denotes a nonlinear differential polynomial $F=I y^{(n)}+Q$ with $I, Q \in \mathcal{u}\{y\}, I Q \neq 0$, and ord $I Q<n$.

1. The case Deg $I=0$.

TheOREM 1. If $I=1$ then $F$ is not sublinear.

Proof. The grading of $u\{y\}$ defined by the sequence $(1+i(d-1) / n)_{i \in N}$ where $d=\operatorname{deg} Q$, is easily seen to be a semi- $F$-grading. By Lemma 4 , there exists an $F$-grading $g$. We suppose $g$ is chosen with maximal order $q$ (so that $0 \leqq q \leqq n-1)$ and use induction on $n-q$ (that is, we suppose the theorem proved for differential polynomials $F$ of order $n$ for which there exists an $F$-grading of order $>q$ ). 
There obviously exists an algebra homomorphism $\gamma: \mathfrak{Q \rightarrow U}\{y\}$ as in the statement of Lemma 8 such that the degree in $\left(y^{(q)}, \cdots, y^{(n-1)}\right)$ of $\gamma\left(Q^{*}\right)$ equals that of $Q^{*}$.

This degree is clearly $>0$. If it is 1 , then the differential equation $y^{(n)}$ $+\gamma\left(Q^{*}\right)=0$ must have a solution which is not an element of $\mathscr{K}[x]$, so that $\left\{y^{(n)}+\gamma\left(Q^{*}\right)\right\}$ does not contain any derivative $y^{(n+t)}$; it follows from Lemma 8 that in this case $F$ is not sublinear. We suppose henceforth that the degree in $\left(y^{(q)}, \cdots, y^{(n-1)}\right)$ of $Q^{*}$ is $\geqq 2$. By Lemma 8 , moreover, in proving the theorem we may replace $F$ by $\gamma\left(F^{*}\right)$, that is, we may suppose that $Q$ is free of each $y^{(i)}$ with $i<q$, that $Q$ has constant coefficients, and that $\operatorname{deg} Q \geqq 2$, and then it is enough to prove that $y^{(n+t)} \notin\{F\}$ for all $t$. By the induction assumption, we may also suppose that there does not exist an $F$-grading of order $>q$.

Now, if we had $y^{(n+t)} \in\{F\}$, it is easy to see that we would have $y^{(n+t-q)}$ $\in\{G\}$, where $G$ is the differential polynomial obtained from $F$ on replacing $\left(y^{(q)}, y^{(q+1)}, \cdots, y^{(n)}\right)$ by $\left(y, y^{\prime}, \cdots, y^{(n-q)}\right)$. Therefore we may replace $F$ by $G$, that is we may suppose that $q=0$. In other words, we may suppose that there does not exist an $F$-grading of order $>0$. By Lemma 4 , then, there does not exist a semi- $F$-grading of order $>0$.

If $r$ is any real number $>w t F$, then the sequence $(1+i / r)_{i \in N}$ defines a grading $h$ of $u\{y\}$ such that if $M=y^{i_{0}} y^{\prime i_{1}} \ldots$ is any monomial appearing in $F$ with nonzero coefficient, then $h(M)=\operatorname{deg} M+r^{-1} w t M<\operatorname{deg} M+1$. Thus every term in $F$ of degree $<d(=\operatorname{deg} Q)$ is of lower grade than every term of degree $d$, and among the terms of degree $d$, those of maximal grade are those of maximal weight. Therefore, by Lemma 2 , there exists a semi- $F$-grading of order equal to the maximal order of any term of $Q$ of degree $d$ and weight equal to wt $Q$. Since there does not exist a semi- $F$-grading of order $>0$, the weight of every term of $Q$ of degree $d$ must be 0 , that is $Q=a y^{d}+R$ where $a \in \mathcal{K}, a \neq 0, R \in \mathcal{K}\{y\}$, ord $R<n$ and $\operatorname{deg} R<d$.

Now, $F$ has a zero $\zeta \neq 0$. If we had $y^{(n+t)} \in\{F\}$, then $\zeta$ would be a zero of $y^{(n+t)}$, that is, we would have $\zeta=c_{0}+c_{1} x+\cdots+c_{s} x^{s}, c_{j} \in \mathcal{K}(0 \leqq j \leqq s), c_{s} \neq 0$, $0 \leqq s<n+t$. By the above, we would have $a \zeta^{d}=-\zeta^{(n)}-R(\zeta)$. The left member of this equation, regarded as an element of the polynomial ring $\Re[x]$, would have degree $s \cdot d$, whereas the right member would have degree $\leqq s(d-1)$. This shows that we cannot have $y^{(n+t)} \in\{F\}$ and completes the proof of the theorem.

2. Second case Deg $I>0$. The following theorem is a partial complement to Theorem 1.

Theorem 2. Suppose $\operatorname{deg} I>0$ and $\operatorname{deg} Q>0$. If there exists an F-grading of order $>$ ord $I$, then $F$ is not sublinear.

Proof. Let there exist an $F$-grading of order $q>$ ord $I$. As in the first part of the proof of Theorem 1, we see we may suppose that the degree of $Q^{*}$ in $\left(y^{(q)}, \cdots, y^{(n-1)}\right)$ is $\geqq 2$. Therefore there exists an algebra homomorphism 
$\gamma: Q \rightarrow \mathcal{U}\{y\}$ as in the statement of Lemma 8 , such that $\operatorname{deg} \gamma\left(Q^{*}\right) \geqq 2$. As ord $I<q, \gamma\left(I^{*}\right) \in \varkappa$. By Theorem $1, \gamma\left(F^{*}\right)$ is not sublinear; in particular, $\left\{\gamma\left(F^{*}\right)\right\}$ does not contain any $y^{(n+t)}$. By Lemma 8 , it follows that $F$ is not sublinear.

Theorem 3. Suppose deg $I>0$. If ord $I=p<n-1$, ord $Q \leqq p, \operatorname{deg}_{y}^{(p)}(Q)$ $\leqq \operatorname{deg}_{y}(p)(I)$, and $I$ and $Q$ do not have a common factor of order $p$, then $F$ is not sublinear.

Proof. Differentiating the congruence $I y^{(n)} \equiv-Q(\bmod [F])$ we find $I y^{(n+1)} \equiv-I^{\prime} y^{(n)}-Q^{\prime}$ so that $I^{2} y^{(n+1)} \equiv-I^{\prime} I y^{(n)}-I Q^{\prime} \equiv I^{\prime} Q-I Q^{\prime}$ $\equiv\left[Q \partial I / \partial y^{(p)}-I \partial Q / \partial y^{(p)}\right] y^{(p+1)}+R_{1}$, where ord $R_{1} \leqq p$; setting $P_{1}=Q \partial I / \partial y^{(p)}$ $-I \partial Q / \partial y^{(p)}$, we see that $P_{1} \neq 0$, for otherwise $I$ and $Q$ would have a common factor of $\operatorname{order} p$. Let $d=\operatorname{deg}_{y}(p) I, q=\operatorname{deg}_{y}{ }^{(p)} P_{1}$ so that $0 \leqq q<\operatorname{deg} I+\operatorname{deg} Q$ $\leqq 2 d$.

We now show that for every natural number $t$ there exists a congruence $I^{t+1} y^{(n+t)} \equiv P_{t} y^{(p+1)^{t}}+R_{t}(\bmod [F])$ where $P_{t} \neq 0$, ord $P_{t} \leqq p, \operatorname{deg}_{y}{ }^{(p)} P_{t}$ $=(t-1)(d-1)+q$, ord $R_{t}<n, \operatorname{deg}_{y}^{(p+1)} R_{t}<t$. For $t=1$, this follows from what has just been proved. Assuming this congruence for a given $t$, we find on differentiating that

$$
\begin{aligned}
& I^{t+1} y^{(n+t+1)} \equiv-(t+1) I^{\prime} I^{t} y^{(n+t)}+P_{t}^{\prime} y^{(p+1)^{t}}+t P_{t} y^{(p+1)^{t-1}} y^{(p+2)}+R_{t}^{\prime}, \\
& I^{t+2} y^{(n+t+1)} \equiv-(t+1) I^{\prime} I^{t+1} y^{(n+t)}+I P_{t}^{\prime} y^{(p+1)^{t}}+t P_{t} y^{(p+1)^{t-1}} I y^{(p+2)}+I R_{t}^{\prime} .
\end{aligned}
$$

Now, $I^{\prime}=\left(\partial I / \partial y^{(p)}\right) y^{(p+1)}+A$, where ord $A \leqq p ; \quad I^{t+1} y^{(n+t)} \equiv P_{t} y^{(p+1)^{t}}+R_{t}$; $P_{t}^{\prime}=\left(\partial P_{t} / \partial y^{(p)}\right) y^{(p+1)}+B$, where ord $B \leqq p ;$ if $p+2=n$ then $I y^{(p+2)} \equiv-Q$, which is of order $<n$ and is free of $y^{(p+1)}$, and if $p+2<n$, then $I y^{(p+2)}$ itself is of order $<n$ and is free of $y^{(p+1)} ; I R_{t}^{\prime}$ is, by similar argument, congruent to a differential polynomial of order $<n$ and of degree $\leqq t$ in $y^{(p+1)}$. Therefore $I^{t+2} y^{(n+t+1)} \equiv\left[-(t+1) \partial I / \partial y^{(p)} P_{t}+I \partial P_{t} / \partial y^{(p)}\right] y^{(p+1)^{t+1}}+R_{t+1}$ where ord $R_{t+1}$ $<n$ and $\operatorname{deg}_{y}{ }^{(p+1)} R_{t+1}<t+1$. Since $\operatorname{deg}_{y}{ }^{(p)} P_{t}=(t-1)(d-1)+q$ and $q<2 d$, it is easy to see that if we set $P_{t+1}=I \partial P_{t} / \partial y^{(p)}-(t+1) P_{t} \partial I / \partial y^{(p)}$ then $P_{t+1} \neq 0$ and $\operatorname{deg}_{y}(p) P_{t+1}=(t-1)(d-1)+q+d-1=t(d-1)+q$. As ord $P_{t+1}$ is obviously $\leqq p$, the proof of the congruence is complete.

That being the case, if $L=\sum_{i=0}^{n+s} a_{i} y^{(i)}$ is any homogeneous linear differential polynomial with $s>0$ and $a_{n+s} \neq 0$, then

$$
\begin{aligned}
I^{s+1} L & =I^{s+1} \sum_{i=0}^{n-1} a_{i} y^{(i)}+\sum_{t=0}^{s} I^{s-t} a_{n+t} I^{t+1} y^{(n+t)} \\
& \equiv I^{s+1} \sum_{i=0}^{n-1} a_{i} y^{(i)}+\sum_{t=0}^{s} I^{s-t} a_{n+t}\left[P_{t} y^{(p+1)^{t}}+R_{t}\right] ; \quad \text { if } s>1,
\end{aligned}
$$

then this is $\equiv a_{s+n} P_{s} y^{(p+1)^{s}}+C(\bmod [F])$ where ord $C<n$ and $C$ is free of $y^{(p+1)^{s}}$. In either case $I^{s+1} L$ is congruent to a nonzero differential polynomial of order $<n$ so that $I^{s+1} L \notin\{F\}: I$. Since a linear differential polynomial of 
order $<n$ cannot be in $\{F\}: I$, as otherwise it would be divisible by $F$, the proof of the theorem is complete.

TheOREM 4. If $I=y^{a}$, where $a$ is a natural number $>0$, and $Q$ is a nonzero homogeneous linear differential polynomial having constant coefficients and order $>0$, then $F$ is not sublinear.

Proof. Write $F=y^{a} y^{(n)}+K$ where $K=\sum_{i=0}^{n-1} c_{i} y^{(i)}$, each $c_{i} \in \mathcal{K}$, and $c_{i} \neq 0$ for some $i>0$ (so that $n>1$ ). It is easy to see that $F$ has a zero $\zeta$ which is not a polynomial in $x$ with constant coefficients. Assuming that there exists a nonzero homogeneous linear differential polynomial $L \in\{F\}: y$, we see that there exist distinct constants $\alpha_{1}, \cdots, \alpha_{r}$, and nonzero elements $\xi_{1}, \cdots, \xi_{r}$ with $\xi_{j}^{\prime}=\alpha_{j} \xi_{j}(1 \leqq j \leqq r)$, and nonzero polynomials $\Theta_{1}, \cdots, \Theta_{r}$ in $x$ with constant coefficients such that $\zeta=\sum_{j=1}^{r} \Theta_{j} \xi_{j}$; because $\zeta$ is not a polynomial in $x$, some $\xi_{j}$ is not a constant, that is, some $\alpha_{j} \neq 0$.

Now

$$
\begin{aligned}
\zeta^{(\nu)} & =\sum_{j=1}^{r}\left(\Theta_{j} \xi_{j}\right)^{(\nu)}=\sum_{j=1}^{r} \sum_{\mu=0}^{\nu}\left(\begin{array}{l}
\nu \\
\mu
\end{array}\right) \Theta_{j}^{(\nu-\mu)} \xi_{j}^{(\mu)}=\sum_{j=1}^{r}\left(\sum_{\mu=0}^{\nu}\left(\begin{array}{l}
\nu \\
\mu
\end{array}\right) \Theta_{j}^{(\nu-\mu)} \alpha_{j}^{\mu}\right) \xi_{j} \\
& =\sum_{j=1}^{r} \Psi_{\nu, j} \xi_{j},
\end{aligned}
$$

where,

$$
\Psi_{\nu, j}=\sum_{\mu=0}^{\nu}\left(\begin{array}{l}
\nu \\
\mu
\end{array}\right) \Theta_{j}^{(\nu-\mu)} \alpha_{j}^{\mu}
$$

is a polynomial in $x$ with constant coefficients, different from 0 for all induces $j$ for which $\alpha_{j} \neq 0$. Therefore

$$
K(\zeta)=\sum_{\nu=0}^{n-1} c_{\nu} \cdot \sum_{j=1}^{r} \Psi_{\nu, j} \xi_{j}=\sum_{j=1}^{r} \mathrm{X}_{j} \xi_{j}, \quad \text { where } \quad \mathrm{X}_{j}=\sum_{\nu=0}^{n-1} c_{\nu} \Psi_{\nu, j}
$$

is a polynomial in $x$ with constant coefficients. Thus,

$$
\begin{aligned}
0=F(\zeta) & =\left(\sum_{j=1}^{r} \Theta_{j} \xi_{j}\right)^{a}\left(\sum_{j=1}^{r} \Psi_{n, j} \xi_{j}\right)+\sum_{k=1}^{r} \mathrm{X}_{k} \xi_{k} \\
& =\sum_{1 \leq i_{1} \leq r,} \cdots, 1 \leq i_{a \leq r, 1 \leq j \leq r} \Theta_{i_{1}} \cdots \Theta_{i_{a}} \Psi_{n, j} \xi_{i_{1}} \cdots \xi_{i_{a}} \xi_{j}+\sum_{k=1}^{r} \mathrm{X}_{k} \xi_{k} .
\end{aligned}
$$

Clearly, $\xi_{i_{1}} \cdots \xi_{i_{a}} \xi_{j}$ is a zero of $y^{\prime}-\left(\alpha_{i_{1}}+\cdots+\alpha_{i_{a}}+\alpha_{j}\right) y$; furthermore, if $\Lambda$ is any set of constants, and if, for each $\lambda \in \Lambda$, $\xi_{\lambda}$ is a nontrivial zero of $y^{\prime}-\lambda y$, then the family $\left(\xi_{\lambda}\right)_{\lambda \in \Delta}$ is linearly independent over the field obtained by adjoining $x$ to the field of constants. Since, in the multiple sum above, the term with set of indices $\left(i_{1}, \cdots, i_{a}, j\right)=(h, \cdots, h, h)$ has coefficient $\Theta_{h}^{a} \psi_{n, h}$, which is $\neq 0$ when $\alpha_{h} \neq 0$, we conclude that, for each index $h$ for which $\alpha_{h} \neq 0$, either there exists a set of indices $\left(i_{1}, \cdots, i_{a}, j\right)$ with 


$$
(a+1) \alpha_{h}=\alpha_{i_{1}}+\cdots+\alpha_{i_{a}}+\alpha_{j},\left(i_{1}, \cdots, i_{a}, j\right) \neq(h, \cdots, h, h),
$$

or else there exists an index $k$ with

$$
(a+1) \alpha_{h}=\alpha_{k} .
$$

As the field generated by $\alpha_{1}, \cdots, \alpha_{r}$ is isomorphic with a field of complex numbers, we may temporarily suppose that each $\alpha_{j}$ is a complex number. Choosing for $h$ an index such that $\left|\alpha_{h}\right|=\sup \left(\left|\alpha_{1}\right|, \cdots,\left|\alpha_{r}\right|\right)$, we see that neither (1) nor (2) can be satisfied. This contradiction completes the proof.

The following lemma is used in the proof of Theorem 5.

Lemma 9. Suppose $\operatorname{deg} I>0$, let $p=$ ord $I$, and let $g$ denote the grading $\left(\alpha_{i}\right)_{i \in N}$ with $\alpha_{i}=0(0 \leqq i \leqq p)$ and $\alpha_{i}=i-p(p<i<\infty)$. If there exists a natural number $k$ with $1 \leqq k \leqq n-p$ such that $F^{*}=\left[\left(I y^{(n-k)}\right)^{(k)}\right]^{*}$ then $F$ is not sublinear.

Proof. The hypothesis implies that $\operatorname{deg} Q>0$ and $g$ is an $F$-grading of order $p$. It is easy to see that for any algebra homomorphism $\gamma: a \rightarrow \mathfrak{u}\{y\}$ as in Lemma 8 , we have

$$
\gamma\left(\left[\sum_{p \leqq v<\infty} y^{(v+1)} \frac{\partial}{\partial y^{(v)}}\right]^{k}\left(I y^{(n-k)}\right)\right)=\left[\gamma(I) y^{(n-k)}\right]^{(k)} .
$$

If $F$ were sublinear there would exist a natural number $s$ such that $y^{(s)}$ $\in\left\{\gamma\left(F^{*}\right)\right\}: \gamma(I)$; choosing $s$ minimal we would have $s>n$, and the equation $\gamma\left(F^{*}\right)=0$ would have a solution $\zeta=c_{0}+\cdots+c_{s-1} x^{s-1}$ with $\zeta \in \mathcal{K}[x]$, and $c_{8-1} \neq 0$; as we would have $\operatorname{deg}_{x} \zeta^{(p)}=s-1-p \geqq n-p>0$ and as $\gamma(I)$ is a polynomial in $y^{(p)}$ of degree $\geqq 1$, we see that we would have $\operatorname{deg}_{x} \gamma(I)(\zeta) \geqq n-p$ and $\operatorname{deg}_{x} \zeta^{(n-k)}=s-1-n+k \geqq k$, and therefore $\operatorname{deg}_{x}\left(\gamma(I)(\zeta) \zeta^{(n-k)}\right) \geqq n-p+k>k$, so that $\gamma\left(F^{*}\right)=\left(\gamma(I) y^{(n-k)}\right)^{(k)}$ could not vanish at $\zeta$.

\section{Chapter III. Derivatives}

In this chapter $F$ denotes an arbitrary nonzero differential polynomial in $\mathcal{u}\{y\}$ of degree $\geqq 2$. The order, separant, and initial of $F$ are denoted by $n, S$, and $I$, respectively.

1. The second derivative. The following theorem shows that the second derivative $F^{\prime \prime}$ is very rarely sublinear.

Theorem 5. Let $F^{\prime \prime}$ be sublinear. Then:

(a) $\operatorname{deg}_{y^{(n)}} F=1$ (so that we may write $F=I y^{(n)}+Q$, with $Q \in \mathcal{U}\{y\}$ and ord $Q<n)$;

(b) $\operatorname{deg} I>0$ (so that $I$ has an order $p$, with $0 \leqq p<n$ ); $Q \neq 0$ and $\operatorname{deg} Q>0$;

(c) there does not exist a semi-F-grading of order $>p$;

(d) the sequence $\left(\alpha_{i}\right)_{i \in N}$, with $\alpha_{i}=0(0 \leqq i \leqq p)$ and $\alpha_{i}=i-p(p<i<\infty)$, is an $F$-grading (of order $p$ ).

Proof. (a) A simple computation shows that $F^{\prime \prime}=\left(S y^{(n+1)}\right)^{\prime}+H y^{(n+1)}+K$, where $H$ and $K$ are of order $\leqq n$. If $\operatorname{deg}_{y}(n) F$ were $\geqq 2$ then we would have 
$\operatorname{deg} S>0$ and ord $S=n$, so that by Lemma 9 , with $(F, p, n, k)$ taken as $\left(F^{\prime \prime}, n, n+2,1\right), F^{\prime \prime}$ would not be sublinear.

(b) If $I$ were of degree 0 then, as $F$ is nonlinear, $Q$ would be nonzero and nonlinear, and by Theorem $1 F$ would not be sublinear, so that $F^{\prime \prime}$ would not be sublinear; if $Q$ were an element of $\mathcal{u}, F^{\prime}=\left(I y^{(n)}\right)^{\prime}+Q^{\prime}$ and by Lemma $9 F^{\prime}$ would not be sublinear, so that $F^{\prime \prime}$ would not.

(c) If there existed a semi- $F$-grading of order $>p$ then by Lemma 4 there would exist an $F$-grading of order $>p$; by Theorem $2, F$ would not be sublinear, so that $F^{\prime \prime}$ would not.

(d) Suppose $\left(\alpha_{i}\right)_{i \in N}$ is not an $F$-grading. Then $g$ is not a semi- $F$-grading, for otherwise by Lemma 2 there would exist a semi- $F$-grading of order $>p$, contradicting (c). Therefore either $g(Q)<g\left(I y^{(n)}\right)$ or ord $Q^{*}<p$. In the latter eventuality we would have $g\left(Q^{*}\right)=0$, that is $g(Q)=0$, whence ord $Q<p$, so that in either case $g(Q)<g\left(I y^{(n)}\right)$. By Lemma 1 then $g\left(Q^{\prime \prime}\right)<g\left(\left(I y^{(n)}\right)^{\prime \prime}\right)$, so that $\left[F^{\prime \prime}\right]^{*}=\left[\left(I y^{(n)}\right)^{\prime \prime}\right]^{*}$. It follows from Lemma 9 that $F^{\prime \prime}$ is not sublinear. This contradiction shows that $g$ is an $F$-grading. Therefore $g(Q)=g\left(I y^{(n)}\right)$ $=n-p>0$, so that ord $Q>p$.

2. Higher derivatives. It is easy to see that if $k$ is a natural number $\geqq 2$ and $F^{(k)}$ is sublinear then $F^{(i)}$ is sublinear $(0 \leqq i \leqq 2)$.

THEOREM 6. Only finitely many derivatives of $F$ can be sublinear.

Proof. Assume the theorem false; then every derivative $F^{(k)}$ of $F$ is sublinear, and we may write $F=I y^{(n)}+Q$ as in Theorem 5 . Writing $p=$ ord $I$ and letting $g$ denote the grading $\left(\alpha_{i}\right)_{i \in N}$ defined in part (d) of Theorem 5 , we see that $g$ is an $F^{(k)}$-grading of order $p$, for every $k$. If $a$ is the algebra over $\mathcal{K}$ generated by the derivatives $y^{(j)}(0 \leqq j<\infty)$ and the coefficients in $F^{*}$, it is easy to see, for any $G \in \mathcal{u}\{y\}$ with ord $G^{*} \geqq p$, that if $G^{*} \in Q$ then $\left[G^{\prime}\right]^{*} \in Q$; therefore $\left[F^{(k)}\right] * \in Q$ for every $k$.

There obviously exists a homomorphism $\gamma: \mathfrak{Q} \rightarrow \mathfrak{U}\{y\}$ as in Lemma 8 such that $\operatorname{deg} \gamma\left(I^{*}\right)>0$; by this lemma each $\gamma\left(\left[F^{(k)}\right]^{*}\right)$ is sublinear. But it is easy to see, for any $G \in \mathcal{u}\{y\}$ with $G^{*}$ of order $\geqq p$ and $G^{*} \in Q$, that $\gamma\left(G^{*}\right)^{\prime}$ $=\gamma\left(\left[G^{\prime}\right]^{*}\right)$. Therefore $\gamma\left(\left(F^{*}\right)^{(k)}\right)=\gamma\left(\left[F^{(k)}\right]^{*}\right)$ for every $k$. Replacing $F$ by $\gamma\left(F^{*}\right)$, we see that we may suppose that $F$ is $g$-homogeneous, $I \in \mathcal{K}\left[y^{(p)}\right]$ and $Q \in \mathcal{K}\left[y^{(p)}, \cdots, y^{(n-1)}\right]$, and $\left\{F^{(i)}\right\}: I$ contains some derivative of $y$. Since we may evidently replace $y^{(p)}, y^{(p+1)}, \cdots, y^{(n)}$ in $F$ by $y, y^{\prime}, \cdots, y^{(n-p)}$, we may also suppose that $p=0$, and therefore that $g$ is the weight grading; then $F$ is isobaric, so that $Q$ is isobaric of weight $n$; of course ord $Q>0$. Also, by Theorem 5 , there does not exist a semi- $F$-grading of order $>0$, and $n \geqq 2$.

Now consider the grading $g^{\prime}=\left(\alpha_{i}^{\prime}\right)_{i \in N}$ with $\alpha_{i}^{\prime}=1+i(0 \leqq i<\infty)$; for any monomial $M, g^{\prime}(M)=\operatorname{deg} M+w t M$. If we had $g^{\prime}\left(I y^{(n)}\right)<g^{\prime}(Q)$ then by Lemma 2 there would exist a semi- $F$-grading of order $>0$, contrary to the above. If we had $g^{\prime}\left(I y^{(n)}\right)>g^{\prime}(Q)$, that is if we had $\operatorname{deg}\left(I y^{(n)}\right)>\operatorname{deg} Q$, then for any polynomial $\zeta=c_{0}+\cdots+c_{r} x^{r} \in \mathcal{K}[x]$ with $c_{r} \neq 0$ and $r>n$ we would 
have $I(\zeta) \zeta^{(n)} \neq 0$ and $\operatorname{deg}_{x} I(\zeta) \zeta^{(n)}=r \operatorname{deg}\left(I y^{(n)}\right)-n>r \operatorname{deg} Q-n \geqq \operatorname{deg}_{x} Q(\zeta)$; therefore $F=I y^{(n)}+Q$ could not have a zero of the form $\zeta$, so that $\{F\}: I$ could not contain any derivative of $y$. It follows that $g^{\prime}\left(I y^{(n)}\right)=g^{\prime}(Q)$, so that $g^{\prime}$ is an $F$-grading, and therefore (see Lemma 1 ) even an $F^{(k)}$-grading for every $k$. By Lemma 8 (with $\gamma$ taken as the identity mapping of $\mathscr{K}\{y\}$ ), we may replace $F$ by its $g^{\prime}$-homogeneous part of highest grade, that is, by its homogeneous part of highest degree. Thus, we may write $F=y^{d-1} y^{(n)}+Q$, where $d>1, Q \in \Re\{y\}, Q \neq 0$, ord $Q<n$, and $Q$ is homogeneous of degree $d$ and isobaric of weight $n$.

The field $\mathscr{K}_{0}$ generated by the coefficients in $F$ is isomorphic with a field of complex numbers; we therefore lose no generality in supposing that $\varkappa_{0}$ is a field of complex numbers.

Since we may replace $F$ by any derivative $F^{(i)}$, we may also suppose that $n>d$. Then we may write

$$
F=y^{d-1} y^{(n)}+\sum_{\nu=1}^{d-1} a_{\nu} y^{d-1-v} y^{\prime \nu} y^{(n-v)}+R,
$$

where $a_{1}, \cdots, a_{d-1} \in \mathcal{K}_{0}, R \in \mathcal{K}_{0}\{y\}$, and each term in $R$ is of degree $\geqq 2$ in $\left(y^{\prime \prime}, \cdots, y^{(n-1)}\right)$. Now, a simple computation shows that

$$
F^{\prime}=y^{d-1} y^{(n+1)}+\sum_{\nu=1}^{d-1} b_{\nu} y^{d-1-v} y^{\prime v} y^{(n+1-\nu)}+R_{\sharp}
$$

where each term in $R \#$ is of degree $\geqq 2$ in $\left(y^{\prime \prime}, \cdots, y^{(n+1)}\right)$, and $b_{1}=d-1+a_{1}$, $b_{\nu}=(d-\nu) a_{\nu-1}+a_{\nu}(2 \leqq \nu \leqq d-1)$. It follows from these equations that if we replace $F$ by a sufficiently high derivative $F^{(j)}$ that each coefficient $a_{\nu}$ will have a real part which is strictly positive. We suppose, then, that this is the case.

We now define for each natural number $t$ an element $Q_{t} \in \mathcal{K}_{0}\{y\}$, homogeneous of degree $d+t$, isobaric of weight $n+t$, of order $<n$, such that $y^{d+t-1} y^{(n+t)}+Q_{t} \equiv 0(\bmod [F])$. Since we obviously may take $Q_{0}=Q$, we suppose that $t>0$ and that $Q_{t-1}$ has already been defined. Differentiating the congruence $y^{d+t-2} y^{(n+t-1)}+Q_{t-1} \equiv 0(\bmod [F])$, and then multiplying by $y$, we find that

$$
\begin{aligned}
& y^{d+t-1} y^{(n+t)}+(d+t-2) y^{\prime} y^{d+t-2} y^{(n+t-1)}+y^{(n)} y \partial Q_{t-1} / \partial y^{(n-1)} \\
& +y \sum_{\nu=2}^{n} y^{(n-v+1)} \partial Q_{t-1} / \partial y^{(n-v)} \equiv 0(\bmod [F]) .
\end{aligned}
$$

Now,

$$
(d+t-2) y^{\prime} y^{d+t-2} y^{n+t-1} \equiv-(d+t-2) y^{\prime} Q_{t-1}(\bmod [F]) .
$$

Also, if $y^{i_{0}} y^{\prime i_{1}} \cdots y^{(n-1) i_{n-1}}$ is a monomial in $Q_{t-1}$, then $\sum_{\nu=0}^{n-1} i_{\nu}=d+t-1$ and $\sum_{\nu=1}^{n-1} \nu i_{\nu}=n+t-1$, so that (subtracting the former sum from the latter) 
$\sum_{\nu=1}^{n-1}(\nu-1) i_{\nu}=n-d+i_{0}$; therefore if this monomial involves $y^{(n-1)}$, that is, if $i_{n-1} \geqq 1$, then $i_{0} \geqq d-2$. Thus, $\partial Q_{t-1} / \partial y^{(n-1)}$ is divisible by $y^{d-2}$, and we may write

$$
\begin{aligned}
y \partial Q_{t-1} / \partial y^{(n-1)} \cdot y^{(n)} & =y^{-d+2} \partial Q_{t-1} / \partial y^{(n-1)} \cdot y^{d-1} y^{(n)} \\
& \equiv-y^{-d+2} \partial Q_{t-1} / \partial y^{(n-1)} \cdot Q(\bmod [F]) .
\end{aligned}
$$

It follows that if we define

$Q_{t}=-(d+t-2) y^{\prime} Q_{t-1}-Q \cdot y^{-d+2} \partial Q_{t-1} / \partial y^{(n-1)}+y \sum_{\nu=1}^{n-1} y^{(n-\nu)} \partial Q_{t-1} / \partial y^{(n-\nu-1)}$, then $Q_{t}$ will have the desired properties.

For each $t$ we may write $Q_{t}=\sum_{\nu=1}^{d-1} a_{t, \nu} y^{d-1-\nu} y^{t+\nu} y^{(n-\nu)}+R_{t}$, where $a_{t, 1}, \cdots, a_{t, d-1} \in K_{0}, R_{t} \in K_{0}\{y\}$, and each term in $R_{t}$ is of degree $\geqq 2$ in $\left(y^{\prime \prime}, \cdots, y^{(n-1)}\right)$. From the recursion formula defining $Q_{t}$ we see, by any easy computation, that

$$
a_{t, \nu}=-a_{\nu} a_{t-1,1}-(t+\nu-1) a_{t-1, \nu}+a_{t-1, \nu+1}, \quad(1 \leqq \nu \leqq d-1),
$$

where $a_{t-1, d}=0$.

Since $y^{(n+s)} \in\{F\}: y$ for some natural number $s>0$, and since ord $Q_{s}<n$, we must have $Q_{s}=0$ for this $s$; in particular, we must have $a_{s, \nu}=0(1 \leqq \nu \leqq d-1)$. On the other hand $a_{0, \nu} \neq 0(1 \leqq \nu \leqq d-1)$, since the real part of each $a_{0, \nu}$ is strictly positive. Therefore there is a natural number $t$ with $1 \leqq t \leqq s$ such that $a_{t, 1}, \cdots, a_{t, d-1}$ are all 0 but $a_{t-1,1}, \cdots, a_{t-1, d-1}$ are not all 0 . Thus, $\left(a_{t-1,1} \cdots, a_{t-1, d-1}\right)$ is a nontrivial solution of the system of homogeneous linear equations

$$
\begin{aligned}
a_{\nu} X_{1}+(t+\nu-1) X_{\nu}-X_{\nu+1} & =0 \quad(1 \leqq \nu \leqq d-2), \\
a_{d-1} X_{1}+(t+d-2) X_{d-1} & =0 .
\end{aligned}
$$

The determinant of this system must therefore vanish. But an easy computation shows that this determinant is equal to

$$
t(t+1) \cdots(t+d-1)+\sum_{\nu=1}^{d-1} \frac{(t+d-1) !}{(t+\nu-1) !} a_{\nu} .
$$

As the real part of each $a_{\nu}$ is positive, this determinant cannot vanish. This contradiction completes the proof.

\section{REFERENCES}

1. E. R. Kolchin, Galois theory of differential fields, Amer. J. Math. vol. 75 (1953) pp. 1-31.

2. J. F. Ritt, Differential algebra, Amer. Math. Soc. Colloquium Publications, vol. 33, New York, 1950.

Columbia University,

NEW YORK, NEW YORK

Pratt Institute,

BROOKLYN, NEW YORK 